\title{
Using New Media for Practice-based Fine Arts Research in the Classroom
}

\author{
Topher Maraffi \\ University of South Carolina Beaufort \\ Beaufort SC \\ cmaraffi@uscb.edu
}

\begin{abstract}
This paper and demo describes our approach and initial results of using David Hockney's work as a model for practice-based arts research in a digital imaging class. Hockney's innovative photocollages of the 1980sdemonstrate an artist thinking in media, while the Hockney-Falco thesis promotes discussion on the relationship artists have with technology. Using Hockney's photocollage work as a practice-based research agenda, we describe a series of class projects to extend his joiner technique into the new dimensions of 3D and interactive media.
\end{abstract}

Practice-based arts research. New media. Pedagogy. David Hockney.

\section{INTRODUCTION}

The British artist David Hockney is referenced in practice-based arts research (Sullivann 2006, Bolt 2006) because his work has not only produced new visual techniques at the intersection of painting and photography (Joyce 1988, Hockney 2001), but has also led toa scientific thesisthat is both innovative and controversial (Falco 2016). In this paper I show that Hockney's working method of continuously engaging technology to solve an aesthetic question makes him a good model for teaching practicebased research in a Fine Arts class on digital media. By using Hockney's novel photo-collage or "joiner" technique and his Secret Knowledge optics work as class discussion topics, art students consider the implications to their own work that technological exploration is an integral part of the artistic process.

In addition to framing Hockney as a practice-based research model, this paper describes how we generalized his methodology to explore his photocollage technique in successive dimensions of media. In a series of projects, students were challenged to build 2D, 3D, and interactive "joiners" using the software tools Photoshop, Blender, and Unity. We used a create and critique process to explore Hockney's cubist concepts of compressing time and space in new media, and then engaged in discussions on the historical relationship visual artists have had with digital imaging tools since the Renaissance. To facilitate thinking in a new digital medium, Hockney's2D joiner method was generalized into research questions that could be applied to any media. We then show initial results of student work in a demo.

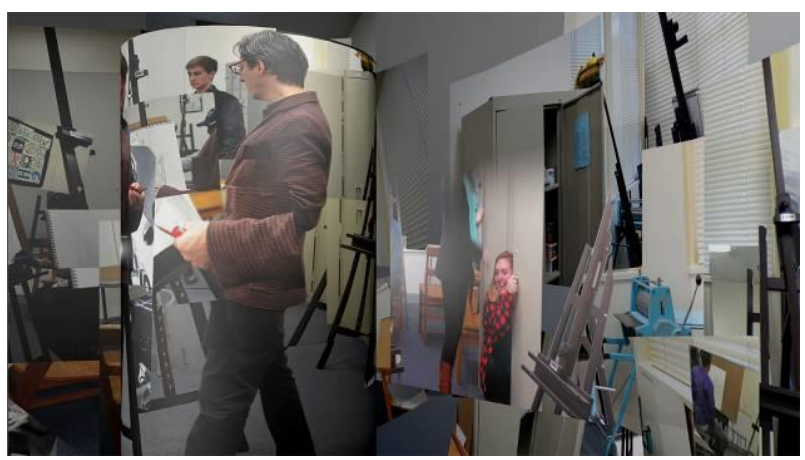

Figure 1: Frame of a 3D animated joiner modelled and rendered in Blender.

\section{HOCKNEY'S PRACTICE AS RESEARCH MODEL}

To introduce my class to the concept of practicebased research in context to their joiner projects, we read two papers that reference Hockney's photography and optics work (Bolt 2006, Sullivan 2006). While Sullivan presents a straight forward argument for practice as research through the process of creating and critiquing artworks, Bolt makes a more subtle argument for changing the relationship artists have with technology to facilitate practice-based pedagogy. Both papers situate practice-based research within the creative process 
of art production while highlighting the differences between theory driven research and theory that emerges from media exploration in the studio.

Sullivan frames art practice as a reflexive process of creating and critiquing artworks as a valid research method that can lead to new knowledge. He likens the art making process as "thinking in a medium", which lends itself to exploring media for possibility spaces that push the boundaries of known techniques. Referencing Pollock's "visual field" as a new way of seeing, artists may change the way we think about and look at art by creating novel aesthetics. Sullivan points to Hockney as an example of an artist that thinks in a medium, by seeing and interpreting what he has done in practice:

\begin{abstract}
"I ask such questions and make the theories only afterwards, not before-only after I have done something. I keep pictures I have done around the studio; you want to look at them. And it takes a while to realize what I really did there, how it works; then I may use that in something else. But though painting can't be done theoretically, all painters must, to a certain extent, analyse their work afterwards." (Hockney 1993)
\end{abstract}

We can compare the art making process to conducting individual experiments using media as a facilitator, where the critique phase interprets the observed results for future work. As such, a body of artwork that attempts to address an aesthetic issue related to a medium, such as Hockney's photo collage work throughout the 1980 s, can be viewed as a series of experiments using the medium itself as a research tool. One difference between such practice-based or "bottoms-up" research and traditional research is that the creativity phase encourages searching the possibility space of a medium for new forms of expression.

\begin{abstract}
"Art practice as research invokes a methodology that is premised on the need to 'create and critique' that opens up the possibility of achieving new understanding...The capacity to look beyond what is merely known to seek the possibility of new understanding is what artists do." (Sullivan 2006).
\end{abstract}

As such, the artistic process has some similarity to creativity in other technical fields where "makers" tinker with known technology to discover unknown applications. For artists, creating novel techniques in a known form of expression requires technical exploration to discover new affordances in their medium. Hockney's exploration of photography in the early 1980s started after he curated an exhibition for the National Gallery for a Series called The Artist's Eye to "get a sense of what it is that an artist notices in artefacts" that others may not notice.
One thing Hockney noticed was that the performative work of the artist was not as visible in photographs as it was in paintings and drawings. This observation led him to a general dissatisfaction with the photographic process, and a critique of the technical limitations of the medium, stating that it was only good for "photographing other pictures" (Hockney 1981). However, rather than discount the medium entirely, Hockney became driven to making the photographic process more like drawing and painting. This tendency to turn an aesthetic problem, mainly observable from a practitioner's point of view, into a technical challenge that must be explored is one of the reasons Hockney is appealing as a model for practice-based research pedagogy.

Turning a critique of a specific artwork into a general problem that drives a practice-based research agenda is a recurring theme in Hockney's work. Instead of discounting photography because of its perceived limitations as a chemical process, he instead spent years working with the medium in an attempt to expand its technical affordances through interdisciplinary experimentation. Hockney's main critique was that the process of capturing a photograph with a camera was one dimensional, with only a moment of artistic input, after which the technological process did the rest. Compared to a painting and drawing, where each mark of the artist records a point of view that takes time and space to both render and read, a photograph lacks similar artistic depth because a camera only captures a single viewpoint (Joyce 1988).

To address the problem of photography, Hockney developed a sophisticated collage technique that in some ways anticipates digital compositing techniques later developed in photo manipulation software like Photoshop. His time-consuming "joiner" technique extended the photographic process through printing, physical collage, and rephotographing the final work, allowing him to insert the artist's hand in the process. In this way, he could use the medium of photography to explore cubist ideas on representing space and perspective, as well as apply expressive composition techniques borrowed from drawing and painting. When we look at a joiner reproduced in print or media, we see a photographic composite that displays a painterly sense of time and narrative space in all the choices Hockney made with both the camera and in physically compositing the resulting prints.

"In Hockney's montages he painted pictures with photographs that severely compressed the picture plane... This is what it is for artists to think in a medium in a way that extends 
discipline knowledge through the mindful use of media." (Sullivan 2006).

We see a similar methodology in the development of Hockney's Secret Knowledge work in the 1990s, when he proposed that the possible widespread use of optical technology in the Renaissance might have significantly pushed western art towards a photographic aesthetic. Once again, Hockney noticed a technical quality in the work of other artists that made him question traditional methods, or at least what was believed by historians to be the working methods of Renaissance artists. In this case, the initial challenge was several drawings by Ingres that displayed a photographic aesthetic and scale. Bolt emphasises that Hockney's hypothesis emerged from his experience as a practitioner:

\begin{abstract}
"It is the special kind of 'sight' that Hockney gained through being a practitioner that enabled him to be able to offer both original and originary approaches and insights into the drawings of Ingres. The specificity of Hockney's experience as an artist, and particularly a drawer, fashioned the nature of the question, the methodology and the types of realisations that emerged from the investigation." (Bolt 2006)
\end{abstract}

Sullivan also emphasizes that Hockney's ideas emerged from a practitioner's desire to know how something was done. He points out that there are a variety of reasons that an artist may study other artists, possibly out of admiration or rivalry, but also to figure out the technicalities of how a particular visual effect may have been done by reading the visual evidence in artwork:

"What is crucial to appreciate is that this controversial assertion arose for Hockney as a consequence of his art making... As a fellow painter, he directed his research at the artefacts themselves, convinced that the visual images held the necessary evidence" (Sullivan 2006).

Hockney believed he saw visual clues in the Ingres drawings, evident in both their line quality and scale that were photographic and pointed to the use of a lens. To a draftsman, technical properties like construction lines and scale matter, because they afford some visual effects while constraining others. Given his background in cross-discipline experimentation, the premise that Ingres might have used optics was a logical result of Hockney's joiner practice. Bolt likens Hockney's ability to make logical connections that non-practitioners would miss to Carter's "material thinking" or Heidegger's "handling":

\footnotetext{
"Material thinking is the logic of practice... his engagement with the tools and technologies of drawing practice produced its own kind of sight or logic... Hockney's research methodology was idiosyncratic and whilst his research findings have been the subject of much debate, it is
}

precisely this idiosyncratic methodology that highlights the importance and relevance of "handling" and "material thinking"." (Bolt 2006).

In his own practice Hockney had no problem with using technology to help him produce a desired visual effect, so it was logical for him to guess that a Renaissance artist would use optical tools if they were available. Critics may claim this is a modern point of view. But the Renaissance was the beginning of modern art practices where professionals competed for commissions, a time when painters were also technologists that made their own media, and as Hockney noted, a time of experimentation with new techniques that displayed an emerging photographic aesthetic. Hockney characterized the likely adoption of optical tools like the camera obscura in the Renaissance as "to see it is to use it." (Hockney 2001)

From his point of view, whether an artist used mirrors and lenses to achieve a new look or saw the photographic quality in other's work and copied it, the aesthetic clearly spread, and those who did use the technology would have been secretive to have an edge on their competition. Technology is a useful tool to a practitioner, whether it is a paintbrush or a camera, and doesn't imply any lack of artistic skill in Hockney's view. If anything, he emphasized that the limitations of the technology required tremendous skill. Elaborate live scenes had to be staged for the optical devices that had precise lighting, and artists must then composite multiple projections of moving subjects into a single image, again with similarities to contemporary digital techniques. But critics continued to view the use of optical drawing aids as a trick or cheat:

\begin{abstract}
"A common response was to question Hockney's motives and to dismiss his claims for an artist with less skill than a European master may have good reason to suggest that such artistic fluency could only be achieved using a visual trick. The late Susan Sontag was reported as saying, "if David Hockney's thesis is correct, it would be a bit like finding out that all the great lovers of history have been using Viagra."... Hockney observed these things because he was able to create connections based on experience and accumulated evidence, and this is at the heart of what research is about. (Sullivan 2006).
\end{abstract}

A good reason to reference Hockney as a practicebased research model is his determination in the face of criticism, which led to a variety of support for his ideas (Falco 2016, Teller 2013). In his own research Hockney was systematic, he constructed a "great wall" of printed images to study photographic aesthetics in Renaissance art, and physically tested the use of optical devices like the camera obscura and camera lucida to see how they would have been used as drawing aids. Much of his work was documented in his Secret 
Knowledge book and BBC television show of the same name (Hockney 2001, 2002, 2006). At a later date, technologist Tim Jenison significantly extended Hockney's initial testing by demonstrating how a Vermeer painting could have been created using lenses and mirrors of the time period (Teller 2013).

His collaboration with optical scientist Charles Falco since 2000resulted in the Hockey-Falco thesis (Hockney 2000, Falco 2016), which provided quantitative experimental support for Hockney's observations of "optical aberrations" in the paintings themselves. Though their thesis generated even more controversy (Boxer 2001), Falco has stated that "this was the most intensive scientific collaboration of my entire career", and garnered a National Art Education Association Ziegfeld Lectureship Award in 2008 for its contribution to art education (Falco 2016).

For this paper, I am less concerned about whether the Hockney-Falco thesis proves correct or not, but instead whether it is useful for teaching practicebased art research in the classroom. Controversial academic debates have a way of making students think, and Hockney's theory and practice imply an exploratory relationship with technology that may be useful for teaching new media. Bolt uses Hockney to argue for a "post-human" pedagogy where artists learn from working with technology, rather than the traditional practice of skills mastery implied by Sontag's comment. Bolt employs Heidegger to recommend a "refiguration" of the artist-technology relationship that is more collaborative, with emergent aesthetic results that can lead to new knowledge:

\begin{abstract}
'We can begin to talk of 'skill with' rather than 'mastery over' technologies, materials and processes... in placing an emphasis on the 'sight' that is gained through handling, Heidegger does not return us to the skills and techniques based programs that preceded the so-called conceptual turn, nor does it re-invoke the purity of formalism. His emphasis on careful and concernful dealings suggests an alternative ethic to mastery and a different engagement from our instrumentalist dealings with tools and materials. It suggests that humans are indebted to and co-responsible for the emergence of art. I would suggest that this refiguration of the human-technology relation sets out principles for a post-human pedagogy. In a digital present this task appears particularly urgent." (Bolt 2006)
\end{abstract}

The way Hockney follows a path of enquiry across traditional boundaries of media and technique makes him appealing for a "post-human" pedagogy. Looking at his body of work as a whole, we can trace a line of research that intimately relates painting and photography, both aesthetically and technologically, from the Renaissance to contemporary digital media. Hockney has stated that we are in a post-photographic age (Hockney 2001), where software tools allow us to return to the forgotten techniques of the old masters, before chemical processes constrained the artistic process to a single viewpoint:

\begin{abstract}
"For 400, nearly 500 years, the hand was involved with the camera. Artists were in it, using the lens. Then for 160 years, you had chemical photography. But that has now come to an end, and with digital photography, you have got the hand back in the camera. This is why photography is changing and actually moving back towards drawing and painting." (Marr 2001).
\end{abstract}

Although he has criticised the Photoshop aesthetic of retouching photos for advertising as "boring" (Hockney 2013), Hockney has embraced digital tools like the iPhone and iPadin his latest work, and is exploring the boundaries of new media and painting (Weschler 2013, Hockney 2015). In each phase of his career since the 1970s, Hockney has collaborated with imaging tools in novel ways that furthered his practice. Some have called this a love-hate relationship with technology (Weschler 2013).But though each new phase may have started with annoyance at being constrained, and then go through a period of obsession, the relationship seems to always end in love. It is this collaborative relationship with technology that makes him an ideal model for a digital media class.

\section{TEACHING PRACTICE-BASED JOINER RESEARCHUSING NEW MEDIA}

I structured my undergraduate Digital Imaging class around exploring Hockney's joiner technique, and used the Hockney-Falco thesis as reference for discussing practice-based art research. The class is taught in a hybrid lecture-lab format using commercial and open-source software on Macintosh computers. The second fundamental media arts class in our Studio Arts bachelors program, students learn how to apply their fine art knowledge to three new media projects: A digital composite using Photoshop, a 3D animation using Blender, and a 3D interactive environment using Unity.

Hockney's consistent engagement with imaging technology while being firmly grounded in fine arts technique seemed a natural fit for our media arts curriculum, where we take a STEAM approach to pedagogy. While STEAM (STEM + Art) has been promoted in the US as a way to use art practice to enhance STEM pedagogy in grade schools (Maeda 2013), the reverse concept (Art + STEM) has an equally important and subtler application in helping 
to change the attitude of fine artists towards technology. I reference STEAM in my media arts classes to emphasize that working with new media is inherently interdisciplinary, requiring artists to address both aesthetic and technical issues that arise in the production of popular media. If we approach Hockney's Secret Knowledge work from this perspective, the way Renaissance artists used lenses runs parallel to the way pre-scientists of the time used them, to enhance their perception and expand their knowledge. No one to my knowledge has suggested that scientists are less competent because of using lenses.

Hockney's work implies that fine artists have been technical since at least the Renaissance, and that innovative practice-based research today requires Bolt's handling of technology as material thinking through media exploration (Bolt 2006). In teaching a digital imaging class to fine artists, we can reframe Renaissance artists as partially being technologists, who developed innovative techniques like chiaroscuro and perspective through experimentation with new technologies like oils paints and lenses, creating the popular media of their times and in the process changing the aesthetics of Western art to be more photographic. The pedagogical goal of my approach is to counteract the prevalent contemporary attitude in fine arts and art history that using technology implies cheating or being less artistically skilled, which may present a psychological barrier for students to engage unfamiliar technology as an integral part of their art making process.

To frame the joiner technique for practice-based arts research in a media arts class, we read about and watched Hockney's process (Joyce 1988, Featherstone 1983), and then had the students create a joiner for their first project. Since it is a digital imaging class, they used digital photography and Photoshop to manipulate images into a 2D composite of a scene we staged in a painting studio. To speed up the process and take advantage of the digital medium, we decided to crowd-source the imagery by staging a group scene of three artists drawing a still life while many photographers walked around and shot the action and space. The wandering photographers were not staged to be invisible in the source material, but are intended to be a performative part of the joiner representation.

At the peak of Hockney's joiner development, he took a week to shoot Pearblossom Highway (Joyce 1988), spending time getting up on ladders to capture undistorted details on signs, contributing to the aesthetic of compress time and space in the final collage. By flattening multiple dimensions into a $2 \mathrm{D}$ representation, it is designed to make you spend more time reading the compressed information, which is more visually interesting:

\begin{abstract}
"Of course, there's no one viewpoint and so I can move things about if I want to. I can bring a tree in closer and things like that. The actual space, from one side to the other, is about five hundred yards, which is a considerable distance... The viewer is forced to wander around in the picture because I was literally wandering around the area." (Joyce 1988).
\end{abstract}

The students were directed to notice details and try to shoot subjects close up and from multiple angles. It is interesting to note that Hockney's joiner process both anticipates how digital textures are shot for mapping 3D models in programs like Blender, as well as how images are often composited in 2D photo editing programs like Photoshop. In addition to crowd-sourcing the imagery, other adjustments to the process were made to emphasize the affordances of the digital medium. Working with virtual photos, rather than prints like Hockney used, did require additional organization of the source material. To build his joiners, Hockney could spread his full-size prints out on a table to physically sort through them, where we had to page through many thumbnail sized images to select one. To make photo selection easier to manage, I pre-sorted the digital photos into categories based on the physical space of the room, with temporality being automatically organized by the time stamps on the files.

Using layers and digital tools in Photoshop, students were encouraged to explore Hockney's joiner technique. Their tasks were to: compose the imagery like they were painting or drawing to represent a narrative of people moving in the space; create a sense of compressed time by overlapping imagery taken throughout the photo shoot; and bend visual lines in the represented space to create multiple points of view. Because Picasso heavily influenced his work, we discussed Hockney's ideas on Cubist perception:

\footnotetext{
"In the art world cubism has been greatly misinterpreted as being about abstraction, which it is not. The late theorizers of cubism never say it is about abstraction, they know it is about perceiving the physical - how we see; what we see... The experience of art is more real, the moment is longer, and we can feel that moment, but in a photograph we can't." (Joyce 1988).
}

I also required them to use some filters and techniques that were specific to Photoshop, which would allow them to think in the medium and explore new possibilities while working in the technique. To help us better understand Hockney's research agenda, we summarized his critique of traditional photography: 
- Unlike painting or drawing, the hand of the artist is not visible in the rendering of the image, only implied in the composition.

- Time is only represented as a fraction of a second, rather than a narrative of many moments, reducing interest for the viewer.

- Space is flattened to one or two points of perspective, instead of being composed of many points of view, which is closer to how we perceive.
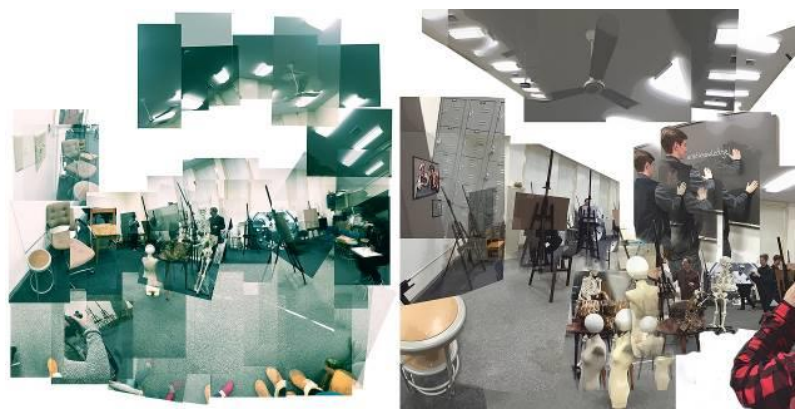

Figure 2: Digital 2D joiners composited in Adobe Photoshop by Basil Hawk and Chloe Threatt.

The first project provided students with a base case for further research. Once the students had presented and critiqued their digital 2D joiners, we began discussing how to extrapolate Hockney's technique to other dimensions in the digital medium, such as in 3D space and 4D time using Blender animation software. To give us a conceptual roadmap of enquiry, we formulated some general research questions from our first experience:

- Can we use digital imaging technology to better represent how people perceive the world, so things are always close and in focus?

- How can we create multiple changing points of view with overlapping moments and perspectives?

- Can we visually compose a non-linear narrative in a photographic medium that is not constrained by realism?

In their second project, the students explored Hockney's research agenda in Blender, an open source 3D animation software program. Extrapolating the joiner technique into a virtual 3D environment that a viewer could move through required the students to go back to their source imagery. First they had to re-construct 2D joiners that could be texture mapped on simple primitives to represent the curvature and depth of the scene. In the animation phase of the project, students had to think about how they could keyframe3D movement in a way that would compress time, and create a multi-dimensional narrative for the viewer. Some of Hockney's experiments in joiner videos (Featherstone 1983, Hockney2012, 2015) were particularly informative, where he overlapped multiple camera footage of a moving subject. We explored similar techniques by rendering multiple camera animations for each 3D scene, and then composited the moving points of view using video layers in Adobe Premiere. The technique did seem to create more visual interest than a render from a single point of view, and can be seen in our demo.

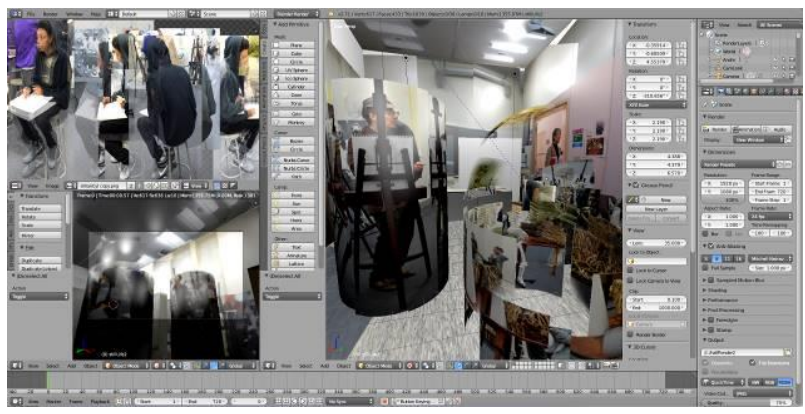

Figure 3: Animated3D joiner modelled in Blender by Hope Sansovich.

In the last phase, students imported the animated 3D environment created in their second project to an open-source game engine called Unity. Video games are the latest imaging technology of the $21^{\text {st }}$ century, encompassing all previous media, with the added $5^{\text {th }}$ dimension of viewer interaction. To explore this new technological medium, artists must embrace the technique of event-based coding. The design of our last practice-based research project was to prototype what a joiner might feel like as an interactive experience. What would a cubist video game be like to play?

First we imported the textured 3D models from our Blender animations to Unity, and then used C\# scripting to design game logics based on Hockney's joiner technique. In a first-person game environment, we employed a standard shooter mechanic to represent the metaphor of shooting photographs in our scene. As players explore the 3D space, they click photos that reveal new details in their environment, representing how we initially explored the 3D space while crowd-sourcing photos for the first project. Instantiating an overlaid camera in the shooting dynamic propels the player into the scene, bringing them temporarily closer to whatever they interact with in the environment. The initial results of simulating some of Hockney's methodology in a game engine are promising, giving us a more formal understanding of the logics behind his techniques. 


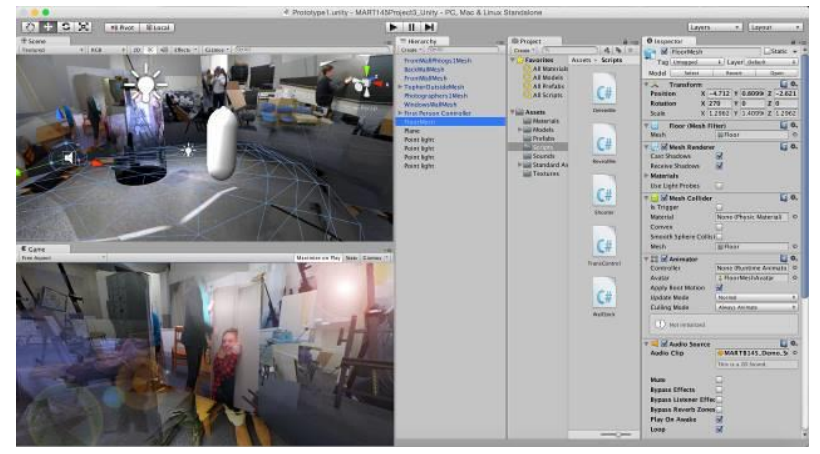

Figure 4: Joiner interactive environment with shooter mechanics designed in Unity game engine.

\section{CONCLUSION}

In this paper we used David Hockney's work as a model for practice-based arts research in a digital imaging class, and describe student projects to extend his joiner technique into new aesthetic dimensions using digital media. I chose Hockney as a model for pedagogy because his working method continuously engages with technology, challenging assumptions made about traditional technique, and his conceptual analysis sparks academic debate across disciplines. We used the "create and critique" process to collaboratively "think in a medium", exploring Hockney's joiner technique in 2D compositing software, a 3D animation program, and a game engine. Our initial results are promising in that they show Hockney's general research questions can be extrapolated to digital mediums with multiple dimensions. As it took Hockney years of intense experimentation to fully develop his 2D joiner technique in the medium of photography, we intend to continue this line of research in future classes. Please view our demo to critique our current results.

Teaching new media to fine art students can be challenging due to the prevailing attitude that art and technology have always been separate endeavours. Relating the subject to the "create and critique" process of practitioners in the canon of art history, including a leading figure in the contemporary art world, challenges those ingrained assumptions. Presenting Hockney's work and thesis in the context of practice-based research did seem to make the students think, and helped to move their art practice away from standard skills learning to a more exploratory relationship with technology. This line of enquiry brings up many interesting points of discussion, including the implication that innovative aesthetic techniques are more likely to emerge from interdisciplinary practice at the boundaries of old and new media, than by exploring either old or new media alone. By providing new media tools to the young artists of today, without losing sight of the collaborative relationship that artists have always had with technology, we can better facilitate the great art of the future.

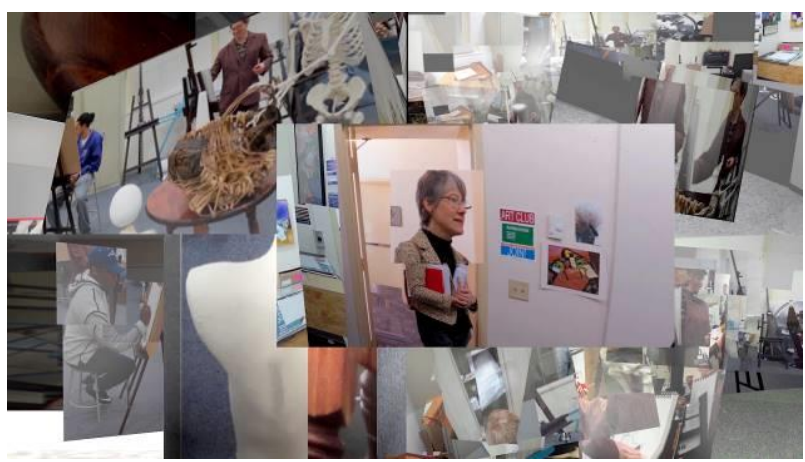

Figure 5: See our demo video to critique the aesthetic results of our practice-based media arts research. Student examples by Shaquiella Holmes, Levi Kinnard, Hope Sansovich, and Chloe Threatt.

\section{REFERENCES}

Bolt, B. (2006) Materializing pedagogies: Working Papers in Art and Design 4, University of Hertford, https://www.herts.ac.uk/ data/assets/pdf file/0015 112381/WPIAAD vol4 bolt.pdf (retrieved 12 March 2016).

Boxer, S. (2001) The Great Optics Debate, New York Times,

http://www.nytimes.com/learning/students/pop/200 11205snapwednesday.html(retrieved 12 March 2016)

Falco, C. (2016) Hockney-Falco Thesis, http://fp.optics.arizona.edu/SSD/artoptics/index.html (retrieved 12 March 2016).

Featherstone, D. (1983) David Hockney: Joiner Photographs, DVD, ArthausMusik, GmbH.

Hockney, D. (2015) Hockney: Live at the Getty, https://www.youtube.com/watch?v=4 Icb28fCz8 (retrieved 12 March 2016).

Hockney, D. (2013) Photoshop is Boring, https://www.youtube.com/watch? $\mathrm{v}=\mathrm{OAx}$ aYGmpoM (retrieved 12 March 2016).

Hockney, D. (2012) Hockney Wolds 9 Cameras, https://www.youtube.com/watch?v=MXSQdMHGsE (retrieved 12 March 2016).

Hockney, D. (2006) Secret Knowledge: Rediscovering the Lost Techniques of the Old Masters (Second Edition) Viking Studio, New York.

Hockney, D. (2002) Secret Knowledge BBC Documentary part 1, YouTube, https://www.youtube.com/watch?v=JKbFZIpNK10 (retrieved 12 March 2016).

Hockney, D. (1981) The artist's eye: Looking at pictures in a book at the National Gallery, July August, 1981. London, UK: The National Gallery. 
Hockney, D., Falco, C. (2000) Optical Insights into Renaissance Art,

http://fp.optics.arizona.edu/SSD/artoptics/papers/OPN.pdf (retrieved 12 March 2016).

Joyce, P. (1988) Hockney On Photography: conversations with Paul Joyce, Harmony Books, New York.

Maeda, J. (2013) STEM + Art = Steam, http://scholarship.claremont.edu/steam/vol1/iss1/34 I (retrieved 12 March 2016)

Marr, A. (2001) What the Eye Didn't See..., The Guardian, 7 October,

http://www.theguardian.com/theobserver/2001/oct/

07/featuresreview.review1 (retrieved 12 March 2016).

Sullivan, G. (2006) Artefacts as Evidence within Changing Contexts: Working Papers in Art and Design 4, University of Hertfordshire, UK

https://www.herts.ac.uk/ data/assets/pdf file/0004 /12397/WPIAAD vol4 sullivan.pdf (retrieved 12 March 2016).

Teller. (2013) Tim's Vermeer, DVD, Sony Pictures Classics, New York.
Vermeer and the Camera Obscura, http://www.essentialvermeer.com/camera obscura/ co one.html\#.VuSKQZMrLwd (retrieved 12 March 2016).

Weiss, P. (2003) Reflections on Art: Scientists debate whether the old masters used optical aids, Science News, Vol. 163, No. 22, 31 May, p. 346. http://www.phschool.com/science/science news/art icles/reflection on art.html (retrieved 12 March 2016).

Weschler, L. (2013) Why David Hockney has a Love-Hate Relationship with Technology, The Smithsonian, USA,

http://www.smithsonianmag.com/arts-culture/whydavid-hockney-has-a-love-hate-relationship-withtechnology-864777/?no-ist (retrieved 12 March 2016).

Weschler, L. (2013) Love Life: David Hockney's Timescapes, YouTube, https://www.youtube.com/watch?v=KyzsY2mXZol (retrieved 12 March 2016). 